\title{
Computing interval-valued reliability measures: application of optimal control methods
}

\author{
Kozin, Igor; Krymsky, Victor
}

Published in:

International Journal of General Systems

Link to article, DOI:

$10.1080 / 03081079.2017 .1294167$

Publication date:

2017

Document Version

Peer reviewed version

Link back to DTU Orbit

Citation (APA):

Kozin, I., \& Krymsky, V. (2017). Computing interval-valued reliability measures: application of optimal control methods. International Journal of General Systems, 46(2), 144-157.

https://doi.org/10.1080/03081079.2017.1294167

\section{General rights}

Copyright and moral rights for the publications made accessible in the public portal are retained by the authors and/or other copyright owners and it is a condition of accessing publications that users recognise and abide by the legal requirements associated with these rights.

- Users may download and print one copy of any publication from the public portal for the purpose of private study or research.

- You may not further distribute the material or use it for any profit-making activity or commercial gain

- You may freely distribute the URL identifying the publication in the public portal

If you believe that this document breaches copyright please contact us providing details, and we will remove access to the work immediately and investigate your claim. 
Computing interval-valued reliability measures: application of optimal control methods

I. Kozine ${ }^{\mathrm{a}}$ and V. Krymsky ${ }^{\mathrm{b}}$

${ }^{a}$ Management Engineering, Technical University of Denmark, Lyngby, Denmark

${ }^{b}$ Ufa State Petroleum Technological University, Russia

I. Kozine is the corresponding author, e-mail: igko@dtu.dk

Kozine, l., \& Krymsky, V. (2017). Computing interval-valued reliability measures: application of optimal control methods. International Journal of General Systems, 46(2), 144-157. DOI: 10.1080/03081079.2017.1294167 


\title{
Computing interval-valued reliability measures: application of optimal control methods
}

\author{
The paper describes an approach to deriving interval-valued reliability measures \\ given partial statistical information on the occurrence of failures. We apply \\ methods of optimal control theory, in particular, Pontryagin's principle of \\ maximum to solve the non-linear optimisation problem and derive the \\ probabilistic interval-valued quantities of interest. It is proven that the \\ optimisation problem can be translated into another problem statement that can \\ be solved on the class of piecewise continuous probability density functions \\ (pdfs). This class often consists of piecewise exponential pdfs which appear as \\ soon as among the constraints there are bounds on a failure rate of a component \\ under consideration. Finding the number of switching points of the piecewise \\ continuous pdfs and their values becomes the focus of the approach described in \\ the paper. Examples are provided.
}

Keywords: imprecise reliability, control theory, Pontryagin’s principle of maximum, bounded failure rate

\section{Introduction}

This paper is yet one more attempt to improve interval-valued reliability characteristics given imprecise or partial information on the occurrence of failures. Imprecise information in our context is antonymous to a point-valued quantity and basically means either an interval or an upper or lower bound for this quantity. Partial statistical information is a probabilistic statement that imposes constraints on the distribution of probability masses over the set of possible outcomes. For example, the probability of event $A$ is as much as two times greater than the probability of event $B$; or the mean time to failure (MTTF) of component $A$ is greater or equal to the MTTF of component $B$. This type of statements reduces the set of possible probability distributions over a possibility set. Complete information about a random value is provided by a precisely known distribution of probabilities over each possible state.

Kozine, I., \& Krymsky, V. (2017). Computing interval-valued reliability measures: application of optimal control methods. International Journal of General Systems, 46(2), 144-157. DOI: 10.1080/03081079.2017.1294167 
There exist a number of models that strive to build upon only available (though imprecise and partial) information avoiding introducing assumptions that are not supported by observation. The reader can find good overviews of such models of uncertainty representation in different sources, for example, in (Walley 1991), (Helton and Oberkampf 2004), (Helton et al. 2010) and (Ferson 2002). The theory of lower and upper previsions, as it was introduced in (Walley 1991) and (Kuznetsov 1991), has served as the theoretical basis for generalising a number of reliability models to interval-valued probability measures. For a brief overview of these models see, for example, (Utkin and Coolen 2007). More specifically, the reliability models of nonreparable systems of general structures (series, parallel and complex connections) generalised to interval-valued probability measures are presented in (Kozine and Filimonov 2001) and (Kozine and Utkin 2005), generalised discrete Markov chains used to model repairable systems are described in (Kozine and Utkin 2002a) and (Skujl 2009), stress-strength models for structural reliability are reported in (Utkin and Kozine 2002, 2010). The theory of lower and upper previsions has been applied to other important issues for reliability and risk analyses like aggregation of imprecise data having different degrees of confidence to different pieces of evidence (Walley 1997) and (Kozine and Utkin 2002b), expert judgement elicitation procedures, and decision making based on imprecise probabilities (Utkin and Augustin 2005, 2007).

In (Kozine and Krymsky 2007) the authors’ belief was that the main obstacle to the practical application of these models is a tangible imprecision in lower and upper probability bounds constructed from a set of imprecise probabilistic pieces of evidence or/and the rapid growth in imprecision that occurs when intervals are propagated through mathematical models.

Kozine, I., \& Krymsky, V. (2017). Computing interval-valued reliability measures: application of optimal control methods. International Journal of General Systems, 46(2), 144-157. DOI: 10.1080/03081079.2017.1294167 
Several attempts have been undertaken to introduce some extra judgements to the set of constraints to limit the set of admissible probability distributions on which a solution is sought and, in this way, to make the bounds tighter. That is, the desire was to remove from the admissible set the distributions that obviously cannot be regarded as reasonable probabilistic models of the phenomena and/or parameters of interest. The results of these studies can be found in (Utkin 2002) and (Kozine and Krymsky 2007, 2009a, 2009b, 2012). The introduction of some of additional constraints complicates the optimisation problem making it nonlinear.

To be able to solve the optimisation problem that becomes non-linear after introducing some meaningful extra constraints another approach was suggested in (Kozine and Krymsky 2007). It consists in employing the calculus of variations. As demonstrated in (Kozine and Krymsky 2007) and then in (Kozine and Krymsky 2009a, 2009b), this way enables to utilise a broader spectrum of statistical judgements, which results in tighter bounds on probability measures. The introduction of direct constraints on probability distributions, like an upper bound on a probability density function (pdf) or/and on the absolute value of its derivative, turned to provide good improvements in precision. This type of constraints is not possible to utilise if Walley's and Kuznetsov's approach (Walley 1991; Kuznetsov 1991) is used in the form of a linear program as a tool for the construction of interval-valued probability measures. In Walley's words this tool is called natural extension.

Despite the obvious improvements in the precision of the constructed measures there is yet one more obstacle on the way of applying the theory of lower and upper previsions to reliability calculations. This obstacle stems from the underlying constraint imposed on the values of random variables. The random variables are bounded, which is rather an unacceptable assumption in reliability modelling. In the cases where time to 
failure is the random variable of interest, which often is, we have to impose an upper bound on it. This bound cannot be known in principle if the system is naturally ageing. That is to say, the necessity to choose this bound imposed by the modelling approach makes the reliability measures rather arbitrary values, as the upper bound is not known and has to be chosen casually.

In (Kozine and Krymsky 2012) the calculus of variations is used again to construct interval-valued probability measures. New constraints are introduced that have relevance for reliability applications. They are upper and lower bounds on failure rate. This has the double effect: better precision in the results and avoidance of the troublesome parameter - the upper bound on time to failure. Another promising outcome of that study was the prospective to depart from the use of rather general and complicated mathematical tool, the calculus of variations. As it was proven, under the constraints on the failure rate, optimal solutions are sought on the class of piecewise exponential distributions. In this way, the problem statement can simply be changed to finding the breaking points at which the probability density function either abruptly jumps up or drops.

Working directly with the class of piecewise exponential distributions may appear computationally easier compared to solving the non-linear optimisation problem. This gives a hope for developing a practical and operational method for constructing interval reliability models based on partial and imprecise data. On this way, we have found a solution to removing one more obstacle to an easier derivation of the solution. In this paper we prove how many switching points a piecewise pdf can have depending on the types of the pieces of information (constraints) we have at hand. To be able to prove this, we have employed methods of optimal control theory (the approach originally suggested in (Krymsky 2014)) and, in particular, Pontryagin’s maximum 
principle (Pontryagin et al. 1962; Hestenes 1966). Adjusting the original problem statement to the problem of control theory, translating it into a problem on the class of piecewise continuous (in particular, piecewise exponential) probability distributions and providing a solution to defining the number of switching points for this class of distribution are the key points described in this paper.

\section{Deriving interval-valued probability measures: problem statement}

Let us formulate a rather general problem of computing bounds $\underline{M}$ and $\bar{M}$ on the expected value of an arbitrary function $g(t)$ over an interval $\left[0, T^{*}\right]$ that is a sample space of a random variable $T$ (time to failure), given the upper, $\bar{a}_{i}=\bar{M}\left(f_{i}(t)\right)$, and lower, $\underline{a}_{i}=\underline{M}\left(f_{i}(t)\right)$, bounds on the expected values of other arbitrary functions $f_{i}(t)$, $i \leq n$. As a particular case, the expected values can be known precisely meaning that for every $i$, the lower and upper bounds are equal to each other. If $f_{i}(t)=t$, the expected value is the first moment. If $f_{i}(t)=t^{2}$, the expected value is the second moment, etc. In case $f_{i}(t)=I_{\left[t_{1}, t_{2}\right]}(t)$, where $I_{\left[t_{1}, t_{2}\right]}(t)$ is an indicator function equal to 1 when $t \in\left[t_{1}, t_{2}\right]$, and equal to 0 otherwise, the expected value is the probability $\operatorname{Pr}\left(T \in\left[t_{1}, t_{2}\right]\right)$.

The problem is stated as follows:

$$
\underline{M}(g)=\inf _{\{\rho(t)\}} \int_{0}^{T^{*}} g(t) \rho(t) d t, \quad \bar{M}(g)=\sup _{\{\rho(t)\}} \int_{0}^{T^{*}} g(t) \rho(t) d t
$$

subject to

$$
\underline{a_{i}} \leq \int_{0}^{T^{*}} f_{i}(t) \rho(t) d t \leq \bar{a}_{i}, \quad i=1,2, \ldots, n, \quad \rho(t) \geq 0, \text { and } \int_{0}^{T^{*}} \rho(t) d t=1,
$$


where $\rho(t)$ is the pdf of a random variable $T$. Here the inf and sup are taken over the set $\{\rho(t)\}$ of all pdfs matching constraints (2). Specifically, each constraint in (2) is associated with a subset of $\{\rho(t)\}$, and the intersection of these subsets, if not empty, defines the admissible set of distributions in which there are pdfs on which solutions of the optimization problems (1)-(2) are found.

As shown in (Kozine and Krymsky 2007), imposing a direct constraint on $\rho(t)$ in the form of an upper bound, $\rho(t) \leq K$, makes the interval $[\underline{M}(g), \bar{M}(g)]$ tighter. An additional improvement is achieved if bounds are imposed on pdf's derivative (Kozine and Krymsky 2009b). A far reaching finding for reliability applications was to assume that an upper bound $\bar{\lambda}$ and a lower bound $\underline{\lambda}$ for a failure rate $\lambda(t)$ are known. That is $\underline{\lambda} \leq \lambda(t) \leq \bar{\lambda}$ (Kozine and Krymsky 2012). Introducing this type of constraints in addition to (2) makes the problem nonlinear; the resulting problem can be solved with the calculus of variations (Kozine and Krymsky 2007, 2009a, 2009b).

In this paper we undertake yet one more attempt to tackle the problem differently. We suggest employing optimal control theory (Pontryagin et al. 1962; Hestenes 1966) to solve the nonlinear program and eventually simplify the derivation of imprecise reliability assessments. We depart from the ideas described in (Krymsky 2014) and derive a new optimization problem focusing on the search of 'switching points' in piecewise pdfs which provide min and max of the objective function, and we demonstrate how this approach may become a bridge to a smaller problem - solving complexity and an increased precision of interval-valued reliability assessments. 


\section{A reliability model based on optimal control theory}

A typical model in control theory describes the relations between 'inputs' (control signals) and 'outputs' (resulted parameters) of a dynamic process. The system dynamics is often described by the differential equations in a state space (Friedland 2005)

$$
d x_{l}(t) / d t=\phi_{l}\left(x_{1}(t), x_{2}(t), \ldots, x_{r}(t), u(t), t\right), l=1,2, \ldots, r
$$

Here $u(t)$ is a control (input) signal, $x_{l}(t), l=1,2, \ldots, r$, are state space coordinates and $\phi_{l}(\bullet), l=1,2, \ldots, r$, are the real-valued functions.

Assume now that $\rho(t)$ is the control substituting $u(t)$ in (3) and $F(t)=$ $\int_{0}^{T^{*}} I_{[0, t]}(y) \rho(y) d y$ is a state space coordinate substituting $x_{1}(t)$ in (3). $F(t)$ is a cumulative distribution function (cdf), and $I_{[0, t]}(y)$ equals 1 if $y \in[0, t]$, or equals 0 otherwise. Such model works if $T^{*} \rightarrow \infty$. Note that in terms of control theory, the situation in which $T^{*} \rightarrow \infty$ is allowed, corresponds to the case of the so-called 'infinite horizon control' (Carlson et al. 1991).

Similarly to (3) we can write

$$
d F(t) / d t=\phi_{1}(F(t), \rho(t), t)=\rho(t) .
$$

In terms of control theory, we need to find the control functions $\rho(t)$ for which the expectation $M(g)$ (1) attains its maximum (minimum) subject to constraints on $\rho(t)$ and its functions. A solution to this 'optimal control problem' can be obtained by applying Pontryagin’s maximum principle (Pontryagin et al. 1962; Hestenes 1966). Let us write the following set of equations (it is assumed that besides constraints (2) the other two, $\rho(t) \leq K$ and $\underline{\lambda} \leq \lambda(t) \leq \bar{\lambda}$, are imposed):

$$
\begin{gathered}
\phi_{0}(F(t), \rho(t), t)=g(t) \rho(t) \\
h_{k}(t)=f_{k}(t) \rho(t) \text { if } k=1,2, \ldots, n \text { or } h_{k}(t)=-f_{k-n}(t) \rho(t)
\end{gathered}
$$




$$
\begin{gathered}
\text { if } k=n+1, n+2, \ldots, 2 n ; h_{2 n+1}(t)=\rho(t), \\
S_{k}=-\underline{a_{k}} \text { if } k=1,2, \ldots, n \text { or } S_{k}=\overline{a_{k}} \text { if } k=n+1, n+2, \ldots, 2 n, \\
\xi_{1}(F(t), \rho(t), t)=\rho(t) ; \xi_{2}(F(t), \rho(t), t)=K-\rho(t), \\
\xi_{3}(F(t), \rho(t), t)=\rho(t) /(1-F(t))-\underline{\lambda}, \xi_{4}(F(t), \rho(t), t)=\bar{\lambda}-\rho(t) /(1-F(t)) .
\end{gathered}
$$

Assume the optimal solution of the formulated problem is $\rho(t)=\rho^{*}(t)$ and, correspondingly, $F(t)=F^{*}(t)$. Then, according to (Hestenes 1966) there exist the following functions $H$ and $L$ :

$$
\begin{gathered}
H(F(t), \rho(t), t, \boldsymbol{\mu})=\psi_{0} \phi_{0}(F(t), \rho(t), t)+\psi_{1}(t) \phi_{1}(F(t), \rho(t), t)+\sum_{k=1}^{2 n+1} \mu_{k} h_{k}(F(t), \rho(t), t) \\
L(F(t), \rho(t), t, \boldsymbol{\mu})=H(F(t), \rho(t), t, \boldsymbol{\mu})+\sum_{j=1}^{4} v_{j}(t) \xi_{j}(F(t), \rho(t), t)
\end{gathered}
$$

in which $\boldsymbol{\mu}=\left(\mu_{1}, \mu_{2}, \ldots, \mu_{2 n+1}\right)$ is the vector of multipliers such that the following relations hold:

(a) The multiplier $\psi_{1}(t)$ is continuous and satisfies the conjugate equation

$$
-d \psi_{1}(t) / d t=\left.(\partial / \partial F) H\left(F(t), \rho^{*}(t), t, \boldsymbol{\mu}\right)\right|_{F(t)=F^{*}(t)} .
$$

In our case $d \psi_{1}(t) / d t=0$, so $\psi_{1}(t)=\psi_{1}=$ const.

(b) The multipliers $\psi_{0}, \mu_{k}, k=1,2, \ldots, 2 n+1$, are also constants; $\psi_{0} \geq 0$ and $\mu_{k} \geq 0, k=1,2, \ldots, 2 n$, with

$$
\mu_{k}\left(\int_{0}^{T^{*}} h_{k}\left(F^{*}(t), \rho^{*}(t), t\right) d t+S_{k}\right)=0, \quad k=1,2, \ldots, 2 n+1
$$

Kozine, I., \& Krymsky, V. (2017). Computing interval-valued reliability measures: application of optimal control methods. International Journal of General Systems, 46(2), 144-157. DOI: 10.1080/03081079.2017.1294167 
(c) The multipliers $v_{j}(t), j=1,2,3,4$, are piecewise continuous over each interval of continuity of $\rho^{*}(t)$. Moreover for each $j=1,2,3,4$ we have

$$
v_{j}(t) \geq 0 \text { and } v_{j}(t) \xi_{j}\left(F^{*}(t), \rho^{*}(t), t\right)=0
$$

The maximum principle is stated by the inequality

$$
H\left(F^{*}(t), \rho^{*}(t), t, \boldsymbol{\mu}\right) \geq H\left(F^{*}(t), \rho(t), t, \boldsymbol{\mu}\right),
$$

which holds for all $\left[F^{*}(t), \rho(t), t\right] \in A$, where $A$ is the set of admissible solutions, i.e., solutions that match the set of constraints. The principle implies that (Hestenes 1966)

$$
\left.(\partial / \partial \rho) L\left(F^{*}(t), \rho(t), t, \boldsymbol{\mu}\right)\right|_{\rho(t)=\rho^{*}(t)}=0
$$

By substituting the expression (8) into (10) and, sequentially, substituting the other variables defined by the above formulas, we obtain the following equality:

$$
\psi_{0} g(t)+\psi_{1}+\sum_{k=1}^{n}\left(\mu_{k}-\mu_{k+n}\right) f_{k}(t)+\mu_{2 n+1}+v_{1}(t)-v_{2}(t)+\frac{v_{3}(t)-v_{4}(t)}{1-F^{*}(t)}=0 .
$$

Meanwhile, the equations (9) show that in each interval of $\rho^{*}(t)$ continuity at least one of the functions $v_{j}(t)$ or $\xi_{j}(F(t), \rho(t), t), j=1,2,3,4$, equals zero. Assume first that $v_{1}(t)=v_{2}(t)=v_{3}(t)=v_{4}(t)=0$, then the expression (11) is reduced to

$$
g(t)=c_{0}+\sum_{k=1}^{n} c_{k} f_{k}(t)
$$

where $c_{0}=-\psi_{1}+\mu_{2 n+1} / \psi_{0} ; c_{k}=-\mu_{k}-\mu_{k+n} / \psi_{0}, k=1,2, \ldots, n$.

The only special case in which the equality (12) is satisfied for reliability applications is when the objective function (1) and the constraints (2) correspond to the values of probabilities at different points of time. For instance, (12) can be satisfied if $n=1, g(t)=I_{\left[0, t^{*}\right]}(t), f_{1}(t)=I_{[0, q]}(t)$ and $q<t^{*}$. The optimisation problem Kozine, I., \& Krymsky, V. (2017). Computing interval-valued reliability measures: application of optimal control methods. International Journal of General Systems, 46(2), 144-157. DOI: 10.1080/03081079.2017.1294167 
corresponding to this case is to find min and max of $M(g)=M\left(I_{\left[0, t^{*}\right]}(t)\right)=F\left(t^{*}\right)$, which can be interpreted as the probability of failure at time $t^{*}$, subject to the known interval-valued probability of failure at time $q$

$$
\underline{a_{1}} \leq \int_{0}^{\infty} f_{1}(t) \rho(t) d t=\int_{0}^{\infty} I_{[0, q]}(t) \rho(t) d t=F(q) \leq \overline{a_{1}} .
$$

Equation (12) is satisfied if $c_{0}=0.5$ and $c_{1}=0.5$, which is a rather trivial case giving us the solution for the probability of failure at time $t^{*}$

$$
\underline{a_{1}} \leq M(g)=M\left(I_{\left[0, t^{*}\right]}(t)\right)=F\left(t^{*}\right) \leq 1 .
$$

Possible location of the curve $y(t)=g(t)-c_{0}-c_{1} f_{1}(t)$ in the Cartesian plane for $c_{0}=0.5 ; c_{1}=0.5$ is shown in Figure 1.

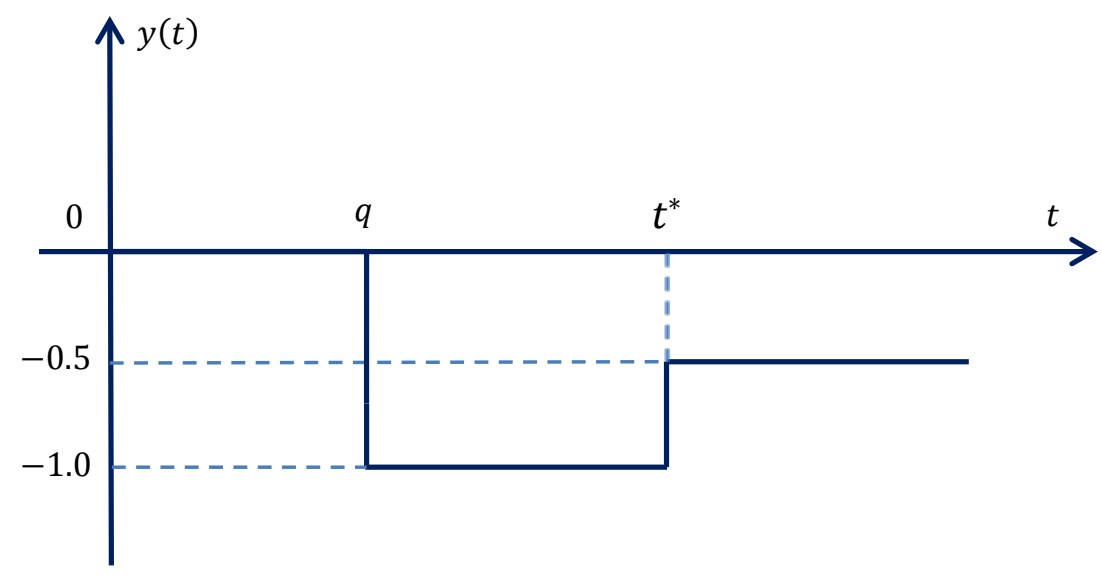

Figure 1. Location of the curve $y(t)=g(t)-c_{0}-c_{1} f_{1}(t)$ in case $g(t)=$ $I_{\left[0, t^{*}\right]}(t)$ and $f_{1}(t)=I_{[0, q]}(t)$

However, a majority of the reliability assessments of interest and available data cannot be expressed as linear combinations of the functions $f_{k}(t), k=1,2, \ldots, n$, so the condition (12) is normally not satisfied. Hence at least one of the functions 
$v_{1}(t), v_{2}(t), v_{3}(t), v_{4}(t)$ in (11) is not equal to zero. If $v_{j}(t) \neq 0$ for any $j=j^{*}$, then the corresponding $\xi_{j^{*}}(F(t), \rho(t), t)$ must be equal to zero in accordance with (9). This means that either $\rho(t)=K$ or a failure rate $\lambda(t)$ attains one of its boundary values $\underline{\lambda}$ or $\bar{\lambda}$. This follows from the expressions (6), (7). As the bounds $\underline{\lambda}$ or $\bar{\lambda}$ are constants, the only probability distribution having as a parameter a constant failure rate is the exponential distribution. Thus we can conclude that the optimal pdf $\rho(t)=\rho^{*}(t)$ is compounded of some intervals of continuity, and at each time interval $\left(t_{i}, t_{i+1}\right)$ either $\rho^{*}(t)=K$ or one of the following two equalities holds: $\rho^{*}(t)=\bar{p}\left(t_{i}\right) \underline{\lambda} \exp (-\underline{\lambda}(t-$ $\left.\left.t_{i}\right)\right)$ or $\rho^{*}(t)=\underline{p}\left(t_{i}\right) \bar{\lambda} \exp \left(-\bar{\lambda}\left(t-t_{i}\right)\right)$. Here $\underline{p}\left(t_{i}\right)$ and $\bar{p}\left(t_{i}\right)$ are the survival probabilities that need to be computed.

\section{Number of continuity intervals of piecewise probability densities}

To estimate the number of continuity intervals for the pdfs at which $M(g)$ attains its maximum or minimum, we rewrite expression (8) by plugging into it expressions (4) and (5):

$$
\begin{gathered}
H\left(F(t), \rho(t), t, \psi_{1}(t), \boldsymbol{\mu}\right)=\psi_{0} g(t) \rho(t)+\psi_{1} \rho(t) \\
+\sum_{k=1}^{n} \mu_{k} f_{k}(t) \rho(t)-\sum_{k=n+1}^{2 n} \mu_{k} f_{k-n}(t) \rho(t)+\mu_{2 n+1} \rho(t)=\gamma(t) \rho(t),
\end{gathered}
$$

where $\gamma(t)=\gamma_{0} g(t)+\gamma_{1} f_{1}(t)+\gamma_{2} f_{2}(t)+\cdots+\gamma_{n} f_{n}(t)+\gamma_{n+1}$, and $\gamma_{0}=\psi_{0} ; \gamma_{1}=\mu_{1}-\mu_{n+1} ; \gamma_{2}=\mu_{2}-\mu_{n+2} ; \ldots ; \gamma_{n}=\mu_{n}-\mu_{2 n} ; \gamma_{n+1}=\psi_{1}+\mu_{2 n+1}$.

In some points $t_{1}, t_{2}, t_{3}, \ldots, t_{Z}$ the function $\gamma(t)$ changes its sign, so it becomes necessary to switch the value of $\rho(t)$ to provide the maximum value of $H\left(F(t), \rho(t), t, \psi_{1}(t), \boldsymbol{\mu}\right)$ according to the maximum principle. In other words, we have 
to select maximum values of $\rho(t)$ if $\gamma(t) \geq 0$ and minimum values of $\rho(t)$ otherwise. This leads us to the conclusion that the number of the continuity intervals of the pdf $\rho^{*}=\rho(t)$ is equal to $Z+1$, where $Z$ is the number of real positive roots of the equation

$$
\gamma_{0} g(t)+\gamma_{1} f_{1}(t)+\gamma_{2} f_{2}(t)+\cdots+\gamma_{n} f_{n}(t)+\gamma_{n+1}=0
$$

In the general case, the value of $Z$ remains unknown. However, in the majority of practical situations, we can easily estimate the maximum number of the positive roots. Namely, if objective function $M(g)$ is a probability and the constraints are imposed on probabilities too (see (1) and (2)), i.e., if $g(t)$ and $f_{i}(t)$ are some indicator functions, then the number of roots $Z$ is known precisely and is equal to 2 . In other cases, where some of the functions $g(t)$ and $f_{i}(t)$ are different from indicator functions, the maximal possible number of positive roots is equal to the order of the polynomial. However, an exact number $Z$ is unknown.

This conclusion gives us the key to understanding the shape of the pdfs for which $M(g)$ attains its lower bound $\underline{M}(g)$ and its upper bound $\bar{M}(g)$.

\section{Examples}

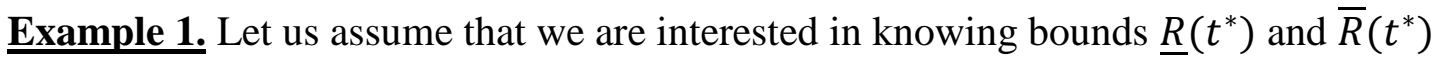
on a system's reliability at time $t^{*}$

$$
R\left(t^{*}\right)=1-\int_{0}^{\infty} I_{\left[0, t^{*}\right]}(t) \rho(t) d t
$$

What is known are the constraints on the failure rate $\underline{\lambda} \leq \lambda(t) \leq \bar{\lambda}$ and the reliability of this system $R(q)$ at another time $q<t^{*}$.

Kozine, I., \& Krymsky, V. (2017). Computing interval-valued reliability measures: application of optimal control methods. International Journal of General Systems, 46(2), 144-157. DOI: 10.1080/03081079.2017.1294167 
This is just a case for which the condition (12) can be satisfied (see also the corresponding Figure 1). The time $t=q$ becomes the 'switching point' between the two continuity intervals of an optimal pdf.

For $0 \leq t<q$ we have an infinite number of admissible pdfs which can provide the reliability $R(q)=1-\int_{0}^{\infty} I_{[0, q]}(t) \rho(t) d t$. For $q \leq t<t^{*}<\infty$ we have to use the boundary values of the failure rate. If we are interested in $\underline{R}\left(t^{*}\right)$, then for $t>q$, $\lambda(t)=\bar{\lambda}$, otherwise $\lambda(t)=\underline{\lambda}$. The shape of $\rho^{*}(t)$ for which $R\left(t^{*}\right)$ attains its minimum is shown in Figure 2.

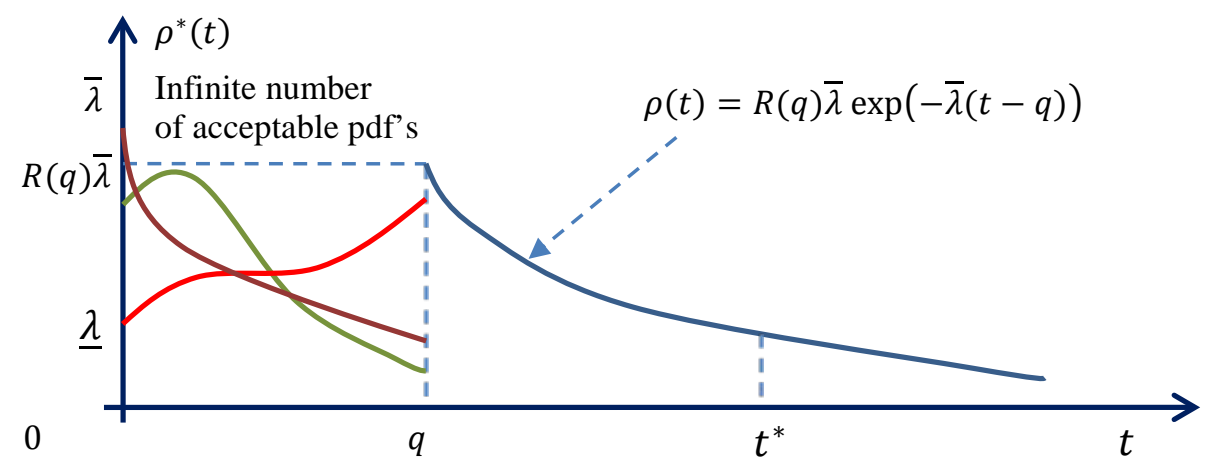

Figure 2. Optimal pdf for which $R\left(t^{*}\right)$ attains its minimum

Finally, $\underline{R}\left(t^{*}\right)=R(q) \exp \left(-\bar{\lambda}\left(t^{*}-q\right)\right) ; \bar{R}\left(t^{*}\right)=R(q) \exp \left(-\underline{\lambda}\left(t^{*}-q\right)\right)$.

Example 2. Now assume that we are interested in knowing the bounds $\underline{\tau}$ and $\bar{\tau}$ on the mean time to failure $\tau=\int_{0}^{\infty} t \rho(t) d t$ given the same constraints as in the previous example: $\underline{\lambda} \leq \lambda(t) \leq \bar{\lambda}$ and the reliability of the system $R(q)$ at time $q$.

In this case we cannot find any continuity interval of $\rho(t)$ for which equality (12) is satisfied. So, the pdfs for which the value of $\tau$ attains its lower or upper bound 
may have the form of either an exponential or a piecewise exponential function with the failure rate $\lambda(t)$ equal either to $\underline{\lambda}$ or $\bar{\lambda}$ within each continuity interval of $\rho(t)$.

Equation (13) here looks as follows:

$$
\gamma(t)=\gamma_{0} g(t)+\gamma_{1} f_{1}(t)+\gamma_{2}=0,
$$

where $g(t)=t, f_{1}(t)=I_{[0, q]}(t)$.

A few possible situations with location of $\gamma(t)$ curve in the Cartesian plane are illustrated in Figure 3, a-d.
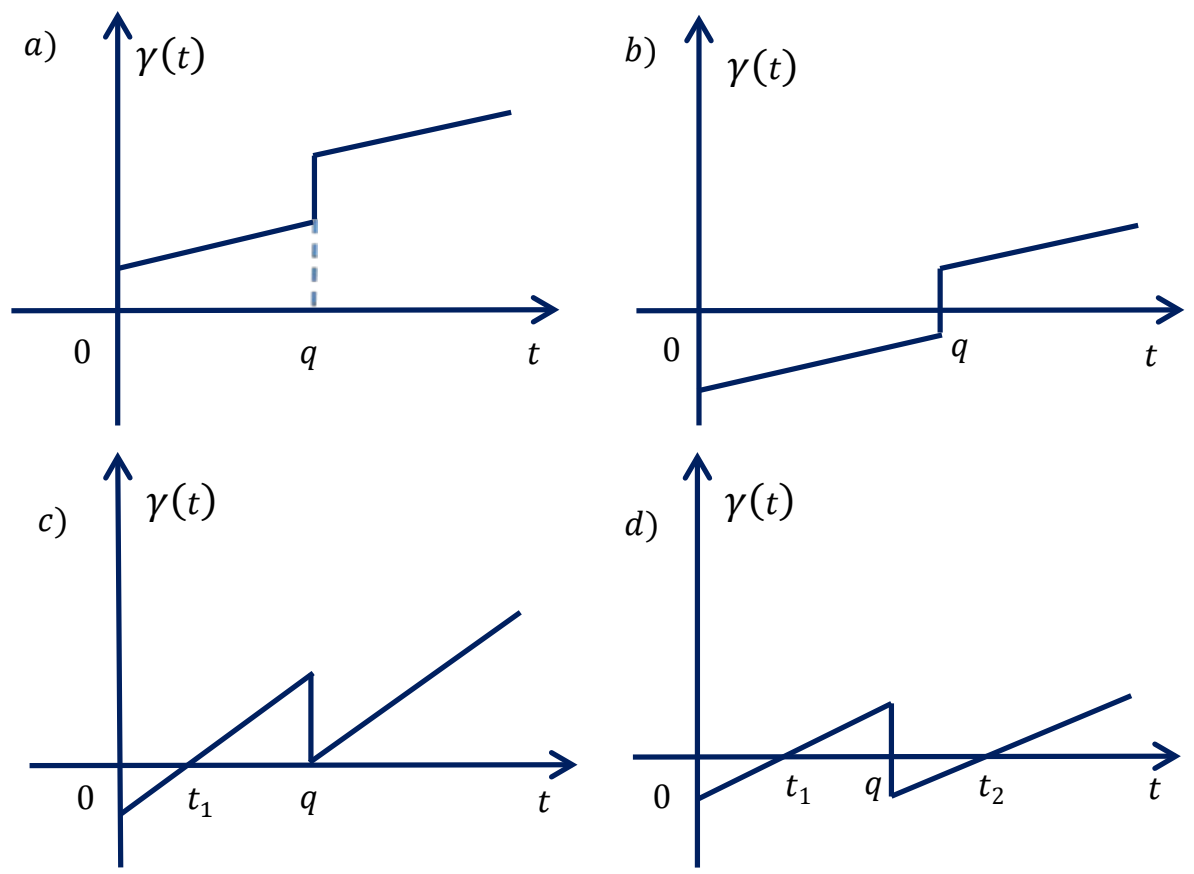

Figure 3. Possible locations of the curve $\gamma(t)=\gamma_{0} g(t)+\gamma_{1} f_{1}(t)+\gamma_{2}$

The situation shown in Figure 3, $a$ takes place if, for instance, $R(q)=\exp (-\bar{\lambda} q)$. The lower bound $\underline{\tau}$ is simply attained on $\rho(t)=\bar{\lambda} \exp (-\bar{\lambda} q)$ without any 'switching points'. So $\underline{\tau}=1 / \bar{\lambda}$.

Kozine, I., \& Krymsky, V. (2017). Computing interval-valued reliability measures: application of optimal control methods. International Journal of General Systems, 46(2), 144-157. DOI: 10.1080/03081079.2017.1294167 
The disposition presented in Figure 3, $b$ corresponds in particular to the following problem statement: we are interested in $\underline{\tau}$ under the following constraint: $R(q)=\exp (-\underline{\lambda} q)$. In this case the optimal pdf takes a piecewise exponential form with one 'switching point' at $t=q$ (Figure 4). The lower bound of the mean time to failure is derived as follows:

$$
\underline{\tau}=\int_{0}^{\infty} P(t) d t=\frac{1}{\underline{\lambda}}\left((1-\exp (-\underline{\lambda} q))+\frac{R(q)}{\bar{\lambda}} .\right.
$$

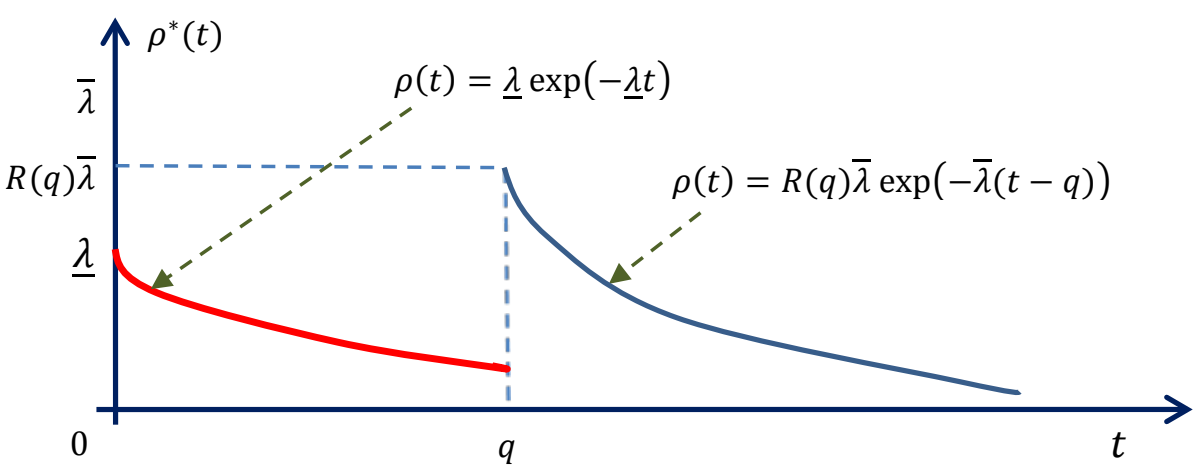

Figure 4: Optimal pdf for which $\tau$ attains its minimum in case $R(q)=\exp (-\underline{\lambda} q)$

The problem becomes more complicated if the following strong inequalities hold:

$\exp (-\underline{\lambda} q)<R(q)<\exp (-\bar{\lambda} q)$. For this case, we may have two or three switching points (figure 3,c,d). Note that one of those switching points corresponds to $t=q$. Let us start from the situation with two switching points (Figure 3,c) and find the value of $t_{1}$ (Figure 5) for the case when we are searching for $\underline{\tau}$.

We have $R(q)=\exp \left(-\bar{\lambda} t_{1}\right) \exp \left(\left(-\underline{\lambda}\left(q-t_{1}\right)\right)\right.$, from which we obtain $t_{1}=-(\ln R(q)+\underline{\lambda} q) /(\bar{\lambda}-\underline{\lambda})$.

Kozine, I., \& Krymsky, V. (2017). Computing interval-valued reliability measures: application of optimal control methods. International Journal of General Systems, 46(2), 144-157. DOI: 10.1080/03081079.2017.1294167 


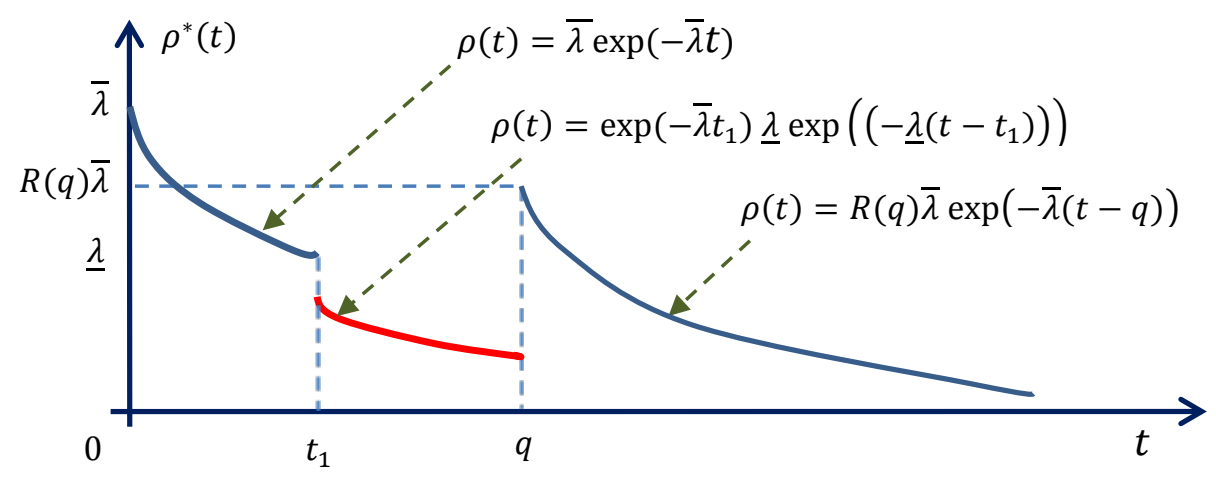

Figure 5. Optimal pdf for which $\tau$ attains its minimum in case $\exp (-\underline{\lambda} q)<R(q)<$ $\exp (-\bar{\lambda} q)$

Hence

$$
\begin{aligned}
\underline{\tau}=\underline{\tau}^{(2)}=\int_{0}^{t_{1}} \exp (-\bar{\lambda} t) d t+\exp \left(-\bar{\lambda} t_{1}\right) \int_{t_{1}}^{q} \exp \left(-\underline{\lambda}\left(t-t_{1}\right)\right) d t \\
\quad+R(q) \int_{t_{1}}^{\infty} \exp (-\underline{\lambda}(t-q)) d t \\
=\frac{\left(1+R(q)-\exp \left(-\bar{\lambda} t_{1}\right)\right)}{\bar{\lambda}}+\frac{1}{\underline{\lambda}}\left(1-\exp \left(-\underline{\lambda}\left(q-t_{1}\right)\right)\right) \exp \left(-\bar{\lambda} t_{1}\right) .
\end{aligned}
$$

Finally, consider the possibility of having three switching points $t_{1}, q, t_{2}$ (Figure 3,d) provided that $\exp (-\underline{\lambda} q)<R(q)<\exp (-\bar{\lambda} q)$. It can be derived that $\underline{\tau}^{(3)}=\underline{\tau}^{(2)}+$ $(\bar{\lambda}-\underline{\lambda}) R(q) \exp \left(-\bar{\lambda}\left(t_{2}-q\right)\right) /(\bar{\lambda} \underline{\lambda})$, and consequently $\underline{\tau}^{(3)}>\underline{\tau}^{(2)}$ for each $t_{2}>q$. This means that the pdf with the two switching points corresponds to the optimal solution.

The derivation of a formula for $\bar{\tau}$ is similar to that demonstrated above for $\underline{\tau}$. The graph of the optimizing pdf for the two switching points is given in Figure 6 . 


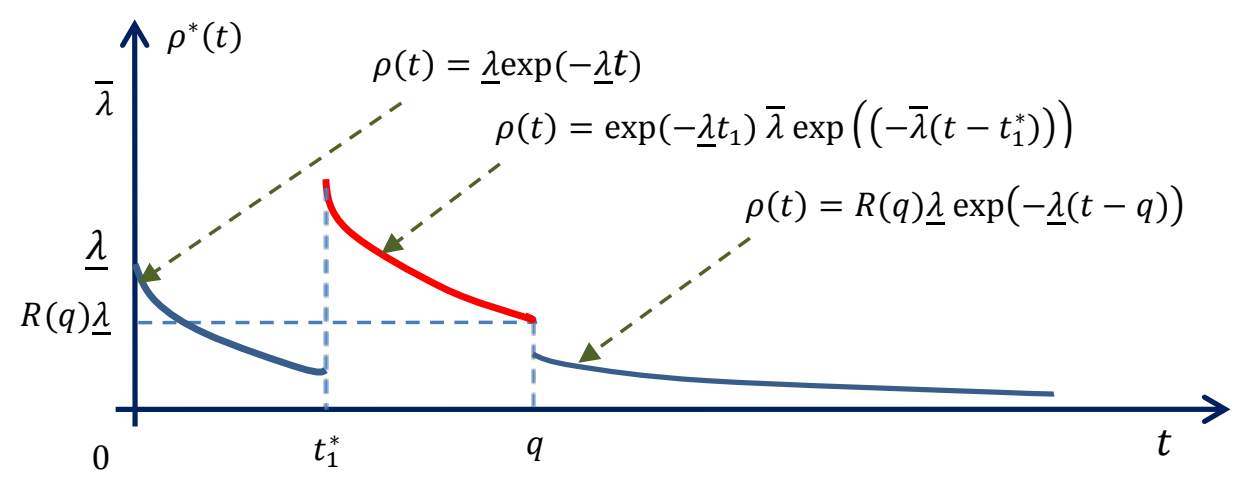

Figure 6. Optimal pdf for which $\tau$ attains its maximum in case $\exp (-\underline{\lambda} q)<R(q)<$ $\exp (-\bar{\lambda} q)$

The switching point can now be obtained as $t_{1}^{*}=(\ln R(q)+\bar{\lambda} q) /(\bar{\lambda}-\underline{\lambda})$.

The upper bound is computed as follows

$$
\bar{\tau}^{(2)}=\frac{1}{\underline{\lambda}}\left(1+R(q)-\exp \left(-\underline{\lambda} t_{1}^{*}\right)\right)+\frac{1}{\bar{\lambda}}\left(1-\exp \left(-\bar{\lambda}\left(q-t_{1}^{*}\right)\right)\right) \exp \left(-\underline{\lambda} t_{1}^{*}\right)
$$

Consider the case of an optimizing pdf with the three switching points. It can be derived that $\bar{\tau}^{(3)}=\bar{\tau}^{(2)}-(\bar{\lambda}-\underline{\lambda}) R(q) \exp \left(-\underline{\lambda}\left(t_{2}-q\right)\right) /(\bar{\lambda} \underline{\lambda})$, and consequently $\bar{\tau}^{(3)}<\bar{\tau}^{(2)}$ for each $t_{2}>q$. So, the pdf with the two switching points just corresponds to the optimal solution. which $M(g)$ attains its lower bound $\underline{M}(g)$ and its upper bound $\bar{M}(g)$.

\section{Problem statement for the class of piecewise exponential distributions}

The two examples in the previous section are special cases for which it was possible to derive analytical expressions for the lower and upper bounds of the reliability characteristics. Perhaps other formulas for reliability applications can be derived assuming that time to failure is governed by a probability distribution confined to the class of piecewise exponential distributions. However, numerical values of the reliability measures of interest can always be derived as a solution to an optimisation 
problem for a multivariate real function that, from the computational point of view, is usually not a difficult problem.

As now the class of the optimizing pdfs is known, we can return to the optimization problems (1)-(2) where pdf $\rho(t)$ explicitly appears in the formulas (Kozine and Krymsky 2012). What is not yet known now are the moments $t_{i}$ at which failure rate $\lambda(t)$ switches from one to the other value $\underline{\lambda}$ and $\bar{\lambda}$. Let us denote the corresponding piecewise exponential pdf as $\operatorname{Exp}\left(\underline{\lambda}, \bar{\lambda}, t_{1}, \ldots, t_{m}, t\right)$.

The integral $\int_{0}^{T^{*}} f_{i}(t) \rho(t) d t$ for the expected value appearing in the constraints (2) as well as $\int_{0}^{T^{*}} g(t) \rho(t) d t$ appearing in (1) can now be rewritten for the case of $T^{*} \rightarrow \infty$ as

$$
\begin{gathered}
\mathrm{G}\left(\underline{\lambda}, \bar{\lambda}, t_{1}, \ldots, t_{m}\right)=\int_{0}^{\infty} g(t) \rho(t) d t=\int_{0}^{\infty} g(t) \operatorname{Exp}\left(\underline{\lambda}, \bar{\lambda}, t_{1}, \ldots, t_{m}, t\right) d t \\
\Phi_{\mathrm{i}}\left(\underline{\lambda}, \bar{\lambda}, t_{1}, \ldots, t_{m}\right)=\int_{0}^{\infty} f_{i}(t) \rho(t) d t=\int_{0}^{\infty} f_{i}(t) \operatorname{Exp}\left(\underline{\lambda}, \bar{\lambda}, t_{1}, \ldots, t_{m}, t\right) d t \\
\mathrm{P}\left(\underline{\lambda}, \bar{\lambda}, t_{1}, \ldots, t_{m}\right)=\int_{0}^{\infty} \rho(t) d t=\int_{0}^{\infty} \operatorname{Exp}\left(\underline{\lambda}, \bar{\lambda}, t_{1}, \ldots, t_{m}, t\right) d t
\end{gathered}
$$

Hence, the reformulated problem statement appears as follows:

$$
\begin{aligned}
& \min _{t_{1}, \ldots, t_{m}} \mathrm{G}\left(\underline{\lambda}, \bar{\lambda}, t_{1}, \ldots, t_{m}\right) \\
& \max _{t_{1}, \ldots, t_{m}} \mathrm{G}\left(\underline{\lambda}, \bar{\lambda}, t_{1}, \ldots, t_{m}\right)
\end{aligned}
$$

subject to

Kozine, I., \& Krymsky, V. (2017). Computing interval-valued reliability measures: application of optimal control methods. International Journal of General Systems, 46(2), 144-157. DOI: 10.1080/03081079.2017.1294167 


$$
\underline{a}_{i} \leq \Phi_{\mathrm{i}}\left(\underline{\lambda}, \bar{\lambda}, t_{1}, \ldots, t_{m}\right) \leq \bar{a}_{i}, i \leq n
$$

and

$$
\mathrm{P}\left(\underline{\lambda}, \bar{\lambda}, t_{1}, \ldots, t_{m}\right)=1
$$

As soon as the functions $g(t)$ and $f_{i}(t)$ are known, all the integrals can be expanded

and the formula for $\operatorname{Exp}\left(\underline{\lambda}, \bar{\lambda}, t_{1}, \ldots, t_{m}, t\right)$ can be explicitly written for all entries, then the optimization problems become manageable and solvable by standard numerical techniques such as gradient methods, simplex-based search methods, genetic algorithms, etc.

\section{Concluding notes}

The constructive finding from the previous study of Kozine and Krymsky (2012) was that possessing bounds on the failure rate of a system limits the class of probability distributions to piecewise exponential distributions. To be more precise, whatever partial and imprecise statistical information on a specific sample set is in possession and, if on top of it bounds on the failure rate are provided, the fitting probability density function belongs to the piecewise exponential class.

The opportunistic benefit of dealing with this class of probability distributions is that the exponential distribution is perhaps the one most frequently used by reliability analysts. It has only one parameter, which is advantageous compared to few-parametric distributions when the samples of failure observations are scarce. It has an easy interpretation: it is simply the average observed number of failures within a certain time interval or, equivalently, the reciprocal of a mean time to failure, which is itself an informative reliability measure. It is also well known that a major part of the lifetime of 
many systems, including human beings, is governed by a failure rate close to being a constant. In technical reliability, this period is called normal operation - in contrast to the wear-in and wear-out (ageing) period, for which the failure rate is clearly timedependent.

Providing maximally and minimally observed number of failures within a certain time interval (often a year) is also feasible and practicable in reliability applications. Furthermore, to compensate for a lack of failure data, it is a normal practice to use generic reliability data sometimes from different sources, some of which can provide one type of measures while some other another type. Given an upper and lower bound on a failure rate are provided, possibly along with other measures, the described approach is an efficient tool to derive the reliability measures of interest.

Except for the above-mentioned positive features of implicating a bounded failure rate there is one more that is of great importance for reliability applications: the sample space for time to failure, on which other reliability measures are derived, stretches from 0 to $\infty$. This is unlike many other imprecise reliability models for which the random variable time to failure is bounded from above by $T^{*}<\infty$. This limitation appears very restrictive for reliability applications, as some reliability models explicitly contain an upper bound on time to failure which is in reality an arbitrary value.

The obtained results call for an exponential-wise approximation of the probability function of time to failure if only partial failure information is available. We believe that the approach described in this paper brings us closer to the practical use of imprecise reliability models.

\section{Acknowledgment}

The authors are grateful to the anonymous reviewer whose scrupulous and valuable suggestions and comments considerably improved the paper. Kozine, I., \& Krymsky, V. (2017). Computing interval-valued reliability measures: application of optimal control methods. International Journal of General Systems, 46(2), 144-157. DOI: 10.1080/03081079.2017.1294167 


\section{References}

Carlson, D.A., Haurie, A.B., and Leizarowitz, A. (1991), Infinite Horizon Optimal Control: Deterministic and Stochastic Systems, Berlin-Heidelberg: Springer Verlag.

Ferson, S. (2002), RAMAS Risk Calc: Risk Assessment with Uncertain Numbers, Boca Raton, FL: Lewis Publishers.

Friedland, B. (2005), Control System Design: An Introduction to State-Space Methods, Mineola, NY: Dover Publications.

Helton, J.C., and Oberkampf, W.L. (2004), 'Alternative Representation of Epistemic Uncertainty', Edited by J.C. Helton and W.L. Oberkampf, Reliability Engineering \& System Safety, 86, 1-3, 1-369.

Helton, J.C., Johnson, J.D., Oberkampf, W.L., and Sallaberry, C.J. (2010),

'Representation of Analysis Results Involving Aleatory and Epistemic Uncertainty’, International Journal of General Systems, 39, 6, 605-646.

Hestenes, M.R. (1966), Calculus of Variations and Optimal Control Theory, New York: John Wiley \& Sons.

Kozine, I.O. and Filimonov, Y.V. (2001), 'Imprecise system reliability’, International Journal of Systems Science, 32, pp. 487-493.

Kozine, I., and Krymsky, V. (2007), Enhancement of natural extension, in 5th International symposium on imprecise probability: Theories and applications (ISIPTA '07), Prague, 16-19 Jul 2007, Prague: Action M Agency, pp. 253-262.

Kozine, I.O., and Krymsky, V.G. (2009a), 'Computing interval-valued statistical characteristics: what is the stumbling block for reliability applications?' International Journal of General Systems, 38, 5, 547-565.

Kozine, I.O., and Krymsky, V.G. (2009b), 'Bounded densities and their derivatives: extension to other domains', Journal of Statistical Theory and Practice, 3, 2538.

Kozine, I.O., and Krymsky, V.G. (2012), ‘An interval-valued reliability model with bounded failure rates’, International Journal of General Systems, 41, 8, pp. 760773.

Kozine, I., \& Krymsky, V. (2017). Computing interval-valued reliability measures: application of optimal control methods. International Journal of General Systems, 46(2), 144-157. DOI: 10.1080/03081079.2017.1294167 
Kozine, I.O, and Utkin, L.V. (2002a), 'Interval-Valued Finite Markov Chains’, Reliable Computing, 8, pp. 97-113.

Kozine, I.O., and Utkin, L.V. (2002b), 'Processing unreliable judgements with an imprecise hierarchical model', Risk Decision Policy, 7 , pp. 1-15.

Kozine, I.O, and Utkin, L.V. (2005), Computing System Reliability Given IntervalValued Characteristics of the Components', Reliable Computing, 11, pp. 19-34.

Krymsky, V.G. (2014), 'Control Theory Based Uncertainty Model for Reliability Applications’, International Journal of Performability Engineering, 10, 5, pp. 477-486.

Kuznetsov, V. (1991), Interval Statistical Models, Moscow: Radio and Sviaz (in Russian).

Pontryagin, L.S., Boltyanskii, V.G., Gamkrelidze, R.V., and Mishchenko, E.F. (1962), The Mathematical Theory of Optimal Processes (trans. by K.N. Trirogoff; ed. by L.W. Neustadt), New York: Interscience Publishers.

Skujl, D. (2009), Discrete time Markov chains with interval probabilities, International Journal of Approximate Reasoning, 50, 8, pp. 1314-1329.

Utkin, L. (2002), 'Imprecise Calculation with the Qualitative Information about Probability Distributions', in Conference on Soft Methods in Probability and Statistics, Heidelberg, New York: Physica-Verlag, pp. 164-169.

Utkin, L.V., and Augustin, Th. (2005), 'Powerful algorithms for decision making under partial prior information and general ambiguity attitudes', in $4^{\text {th }}$ International Symposium on Imprecise Probabilities and Their Applications, ISIPTA'05, July, Pittsburg, USA, pp. 349-358.

Utkin, L.V., and Augustin, Th. (2007), 'Decision making under incomplete data using the imprecise Dirichlet model', International Journal of Approximate Reasoning. 44, 3, 322-338.

Utkin, L.V., and Coolen, F. (2007), 'Imprecise Reliability: an Introductory Overview', Computational Intelligence in Reliability Engineering (SCI), 40, 261-306.

Kozine, I., \& Krymsky, V. (2017). Computing interval-valued reliability measures: application of optimal control methods. International Journal of General Systems, 46(2), 144-157. DOI: 10.1080/03081079.2017.1294167 
Utkin L.V., and Kozine, I.O. (2001), 'Different Faces of the Natural Extension’, in $2^{\text {nd }}$ International Symposium on Imprecise Probabilities and Their Applications ISIPTA’01, Ithaca, NY: Shaker, pp. 316-323.

Utkin, L., and Kozine, I. (2002), 'Stress-Strength Reliability Models under Incomplete Information', International Journal of General Systems, 31, 6, 549-568.

Utkin, L., and Kozine, I. (2010), 'On new cautious structural reliability models in the framework of imprecise probabilities', Structural Safety, 32, 411-416.

Walley, P. (1991), Statistical Reasoning with Imprecise Probabilities, New York: Chapman and Hall.

Walley, P. (1997), Statistical inferences based on a second-order possibility distribution’, International Journal of General Systems, 9, 337-383.

Kozine, I., \& Krymsky, V. (2017). Computing interval-valued reliability measures: application of optimal control methods. International Journal of General Systems, 46(2), 144-157. DOI: 10.1080/03081079.2017.1294167 